Supplement of Solid Earth, 11, 1551-1569, 2020

https://doi.org/10.5194/se-11-1551-2020-supplement

(C) Author(s) 2020. This work is distributed under

the Creative Commons Attribution 4.0 License.

(c) (1)

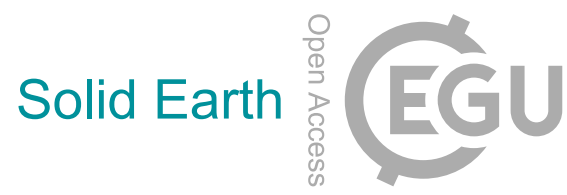

Supplement of

\title{
Increased density of large low-velocity provinces recovered by seismologically constrained gravity inversion
}

Wolfgang Szwillus et al.

Correspondence to: Wolfgang Szwillus (wolfgang.szwillus@ifg.uni-kiel.de)

The copyright of individual parts of the supplement might differ from the CC BY 4.0 License. 


\section{Conversion factor inversion}

We estimate a depth dependent velocity-density conversion factor $p(z)$ using the quantities derived in the main text. Each potential density anomaly is assigned a constant velocity anomaly equal to the mean of SMEAN2 over the area of the density anomaly. This velocity anomaly is multiplied with the reference density from the adiabatic density model (described in the 5 Appendix of the manuscript), giving for each anomaly a density $\bar{\rho}$ it would have, if the conversion factor $p=1$. We calculate a rescaled forward calculation matrix $\bar{A}$, which gives the gravity effect of each potential density anomaly assuming $p=1$.

$\bar{A}_{i k}=A_{i k} \bar{\rho}_{k}$

Next, we sum the gravity effect of all potential density anomalies at the same depth, to obtain matrix $B$ :

$$
B_{i j}=\sum_{k \in \mathcal{D}_{\mid}} \bar{A}_{i k}
$$

where $\mathcal{D}_{j}$ is the collection of indices of potential density anomalies at depth $j$.

The vector of optimal depth-dependent conversion factors $\hat{\mathbf{p}}$ should minimize the following least-squares functional:

$\chi(\mathbf{p})=\|B \mathbf{p}-\mathbf{g}\|_{\Sigma_{g}^{-1}}^{2}+\beta\|\mathbf{p}\|^{2}+\gamma\|D \mathbf{p}\|^{2}$.

Here, $\beta$ and $\gamma$ are two regularization parameters that control the dampening and smoothing of the solution. $D$ is the finitedifference matrix to calculate the first depth derivative of $\mathbf{p}$. The minimizing $\hat{\mathbf{p}}$ is:

$15 \hat{\mathbf{p}}=\left(B^{T} \Sigma_{g}^{-1} B+\beta I+\gamma D^{T} D\right)^{-1} B^{T} \Sigma_{g}^{-1} \mathbf{g}$.

$\beta$ and $\gamma$ are the critical parameters that control the quality of the solution.

We apply this technique to the isostatic residual and find that the results are mainly determined by whichever of $\beta$ and $\gamma$ is largest. If the regularization is weak, the resulting $\mathbf{p}$ are unrealistically oscillatory. Our preferred solution uses $\beta=10^{3}, \gamma=10^{4}$ (Fig. S1). The conversion factor is quite similar to Fig. 9a from the main manuscript, although the amplitude of the conversion factor is more subdued.

However, even with very little regularization, a depth-dependent conversion factor is only able to explain the gravity data to a very limited degree. Since the RMS value of the isostatic residual is around $17 \mathrm{mGal}$, the remaining misfit of $>14 \mathrm{mGal}$ implies a variance reduction of less than $30 \%$. For this reason we did not pursue this approach further and instead allowed an unconstrained density inversion without a velocity-density conversion. 

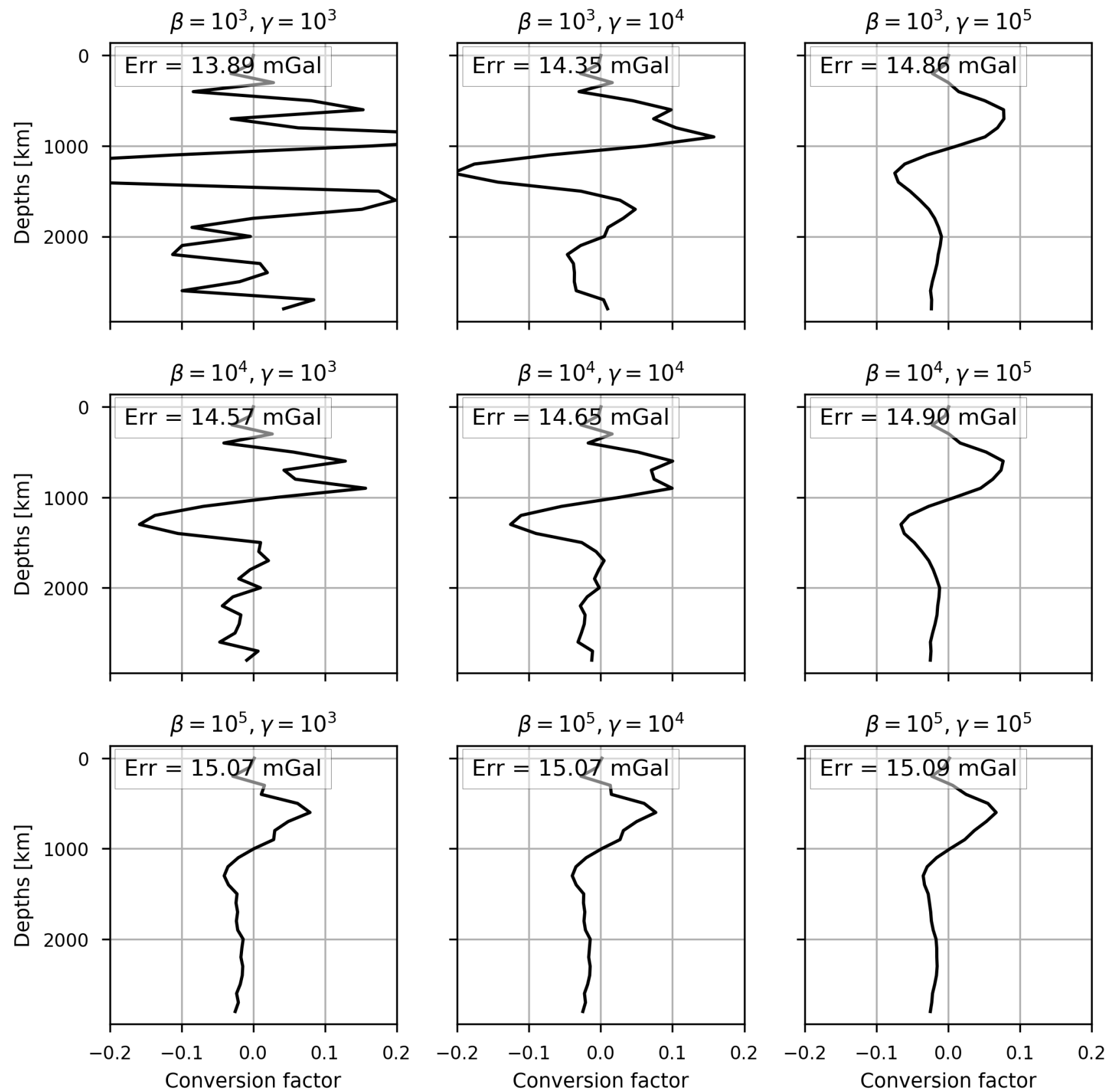

Figure S1. Inverted velocity to density scaling factor for different amounts of regularization. $\gamma$ increases from left to right and $\beta$ increases from top to bottom. The inset error is the RMS of the difference with respect to the isostatic residual. Our preferred model is $\beta=10^{3}$ and $\gamma=10^{4}$. 